\title{
Dynamical symmetry breaking in gauge-Higgs unification on an orbifold
}

\author{
Naoyuki Haba, ${ }^{1}$ Yutaka Hosotani, ${ }^{2}$ Yoshiharu Kawamura, ${ }^{3}$ and Toshifumi Yamashita ${ }^{4}$ \\ ${ }^{1}$ Institute of Theoretical Physics, University of Tokushima, Tokushima 770-8502, Japan \\ ${ }^{2}$ Department of Physics, Osaka University, Toyonaka, Osaka 560-0043, Japan \\ ${ }^{3}$ Department of Physics, Shinshu University, Matsumoto, Nagano 390-8621, Japan \\ ${ }^{4}$ Department of Physics, Kyoto University, Kyoto, 606-8502, Japan
}

(Received 2 February 2004; published 30 July 2004)

\begin{abstract}
We study the dynamical symmetry breaking in the gauge-Higgs unification of the five-dimensional theory compactified on an orbifold, $S^{1} / Z_{2}$. This theory identifies Wilson line degrees of freedom as "Higgs doublets." We consider $S U(3)_{c} \times S U(3)_{W}$ and $S U(6)$ models with the compactification scale of order of a few TeV. The gauge symmetries are reduced to $S U(3)_{c} \times S U(2)_{L} \times U(1)_{Y}$ and $S U(3)_{c} \times S U(2)_{L} \times U(1)_{Y} \times U(1)$, respectively, through the orbifolding boundary conditions. We estimate the one loop effective potential of "Higgs doublets," and find that the electroweak breaking is realized through the radiative corrections when there are suitable numbers of bulk fields possessing the suitable representations. The masses of "Higgs doublets" are $\mathcal{O}(100) \mathrm{GeV}$ in this scenario.
\end{abstract}

DOI: 10.1103/PhysRevD.70.015010

PACS number(s): 11.25.Mj, 12.10.Dm, 12.60.Cn, 12.60.Fr

\section{INTRODUCTION}

Much attentions have been paid to gauge theories in higher dimensions [1-5]. [See, for examples, grand unified theories (GUTs) in higher dimensions on orbifolds.] One of the strongest motivations of the higher dimensional gauge theory is based on the very attractive idea that the gauge and the Higgs fields can be unified in higher dimensions [6,7]. Recently, this possibility has been revisited in Refs. $[8-15,26]$. In these scenarios the Higgs doublets are identified with the extra-dimensional components of the gauge fields in higher dimensions. The masses of "Higgs fields" are forbidden by the higher dimensional gauge invariance. This is the reason why the "Higgs fields" have at most only finite masses of the order of the compactification scale in these scenarios. The gauge group in higher dimensions must be larger than the standard model (SM) gauge group in order to obtain the "Higgs doublets" from the gauge fields in higher dimensions. The gauge symmetries are reduced by the orbifolding boundary conditions of extra dimensions. The identification of "Higgs fields" as a part of gauge supermultiplet has been considered in five-dimensional (5D) $N$ $=1$ supersymmetric (SUSY) gauge theory whose fifth coordinate is compactified on the $S^{1} / Z_{2}$ orbifold [9-13], which corresponds to the four-dimensional (4D) $N=2$ SUSY gauge theory. Also it is considered in six-dimensional (6D) $N=2$ SUSY gauge theory, whose fifth and sixth coordinates are compactified on the $T^{2} /\left(Z_{2} \times Z_{2}^{\prime}\right)$ orbifold [10], which corresponds to the $4 \mathrm{D} N=4$ SUSY gauge theory.

In this paper, we consider 5D non-SUSY and $N=1$ SUSY theories compactified on an orbifold, $S^{1} / Z_{2}$, where Wilson line degrees of freedom are identified as "Higgs doublets." Quarks and leptons are assumed to be localized on the 4D wall. We consider $S U(3)_{c} \times S U(3)_{W}$ and $S U(6)$ models with the compactification scale being a few $\mathrm{TeV}$. The gauge symmetries are reduced to $S U(3)_{c} \times S U(2)_{L} \times U(1)_{Y}$ and $S U(3)_{c} \times S U(2)_{L} \times U(1)_{Y} \times U(1)$, respectively, through the orbifolding boundary conditions. We estimate the one loop effective potential of "Higgs doublets," and find that the electroweak breaking is realized through the radiative corrections when there are suitable numbers of bulk fields possessing the suitable representations. The masses of "Higgs doublets" are $\mathcal{O}(100) \mathrm{GeV}$ in this scenario. The suitable value of $\sin ^{2} \theta_{W}$ and the gauge coupling unification are assumed to be realized by the effects of wall-localized kinetic terms, which may not respect the bulk symmetry. ${ }^{1}$ We should also assume the baryon number symmetry to avoid rapid proton decay in the $\mathrm{TeV}$ scale compactification.

\section{GAUGE-HIGGS UNIFICATION ON $S^{1} / Z_{2}$}

We will consider 5D $S U(N)$ gauge theory on $S^{1} / Z_{2}$ $\otimes M^{4}$. The gauge fields propagate in the bulk. The fifth dimensional coordinate $(y)$ is assumed to be compactified on an $S^{1} / Z_{2}$ orbifold. Under the parity transformation of $Z_{2}$ which transforms $y \rightarrow-y$, the gauge field $A_{M}\left(x^{\mu}, y\right)[M$ $=\mu(=0-3), 5]$ in the $5 \mathrm{D}$ space-time transforms as

$$
\begin{aligned}
& A_{\mu}\left(x^{\mu},-y\right)=P A_{\mu}\left(x^{\mu}, y\right) P^{\dagger}, \\
& A_{5}\left(x^{\mu},-y\right)=-P A_{5}\left(x^{\mu}, y\right) P^{\dagger},
\end{aligned}
$$

where $P$ is the operator of $Z_{2}$ transformation. Two walls at $y=0$ and $\pi R$ are fixed points under $Z_{2}$ transformation. The physical space can be taken to $0 \leqslant y \leqslant \pi R$. Considering the $S^{1}$ boundary condition,

$$
A_{M}\left(x^{\mu}, y+2 \pi R\right)=T A_{M}\left(x^{\mu}, y\right) T^{\dagger},
$$

the reflection around $y=\pi R, Z_{2}^{\prime}$, is given by

$$
P^{\prime}=T P .
$$

The gauge field $A_{M}\left(x^{\mu}, y\right)$ transforms

\footnotetext{
${ }^{1}$ For other possibilities, the power law unification [16] or the accelerated unification [17] might be useful.
} 


$$
\begin{aligned}
& A_{\mu}\left(x^{\mu}, \pi R-y\right)=P^{\prime} A_{\mu}\left(x^{\mu}, \pi R+y\right) P^{\prime \dagger}, \\
& A_{5}\left(x^{\mu}, \pi R-y\right)=-P^{\prime} A_{5}\left(x^{\mu}, \pi R+y\right) P^{\prime \dagger} .
\end{aligned}
$$

under the parity transformation of $Z_{2}^{\prime}$. It should be noticed that the signs of parities of $A_{5}$ are opposite to those of $A_{\mu}$. According to eigenvalues, $( \pm, \pm)$, of parities, $\left(P, P^{\prime}\right)$, the field $A_{\mu}\left(x^{\mu}, y\right)$ is divided into four eigenfunctions as

$$
\begin{aligned}
& A_{\mu}\left(x^{\mu}, y\right)_{(+,+)}=\frac{1}{\sqrt{2^{\delta_{n, 0}} \pi R}} \sum_{n=0}^{\infty} A_{\mu}^{(n)}\left(x^{\mu}\right)_{(+,+)} \cos \left(\frac{n y}{R}\right), \\
& A_{\mu}\left(x^{\mu}, y\right)_{(+,-)}=\frac{1}{\sqrt{\pi R}} \sum_{n=0}^{\infty} A_{\mu}^{(n)}\left(x^{\mu}\right)_{(+,-)} \cos \left(\frac{(n+1 / 2) y}{R}\right),
\end{aligned}
$$

$$
A_{\mu}\left(x^{\mu}, y\right)_{(-,+)}=\frac{1}{\sqrt{\pi R}} \sum_{n=0}^{\infty} A_{\mu}^{(n)}\left(x^{\mu}\right)_{(-,+)} \sin \left(\frac{(n+1 / 2) y}{R}\right) \text {, }
$$

$$
A_{\mu}\left(x^{\mu}, y\right)_{(-,-)}=\frac{1}{\sqrt{\pi R}} \sum_{n=0}^{\infty} A_{\mu}^{(n)}\left(x^{\mu}\right)_{(-,-)} \sin \left(\frac{(n+1) y}{R}\right) .
$$

The expansion of $A_{5}\left(x^{\mu}, y\right)$ is done in the same way. The parity eigenvalue of the field $A_{5}\left(x^{\mu}, y\right)$, is opposite to that of $A_{\mu}$. The massless states surviving in the low energy are zero mode components with parity transformation $\left(P, P^{\prime}\right)=(+$, $+)$. This paper will consider the situation that zero modes of $A_{5}$ are $(\mathbf{1}, \mathbf{2}, 1 / 2)$ or $(\mathbf{1}, \mathbf{2},-1 / 2)$ under $S U(3)_{c} \times S U(2)_{L}$ $\times U(1)_{Y}$. We regard these components as "Higgs doublets," then we call this theory gauge-Higgs unification. Here the local gauge invariance in the 5D guarantees the masslessness of the "Higgs field," so the Higgs mass should be finite after the radiative corrections. We will study two models in the following sections. In Sec. III, we consider $S U(3)_{c}$ $\times S U(3)_{W}$ gauge theory, where the nontrivial parity operators, $P=P^{\prime}=\operatorname{diag}(1,1,-1)$ realizes the gauge reduction of $S U(3)_{W} \rightarrow S U(2)_{L} \times U(1)_{Y}$ as well as the "Higgs doublets" appear as the zero modes in $A_{5}$ [9-11]. In Sec. IV, we will consider the 5D $S U(6)$ theory with the $Z_{2}$ parity operators, $P=\operatorname{diag}(1,1,1,1,-1,-1)$ and $P^{\prime}=\operatorname{diag}(1,-1,-1,-1,-1$, $-1)[10,11]$. In both models, we estimate the one loop effective potential of the "Higgs doublets" including the effects of Kaluza-Klein (KK) [18] modes and bulk matter fields. And we study the vacuum structure of the models and calculate the mass of the "Higgs fields." We will also study the SUSY version, where $A_{5}$ becomes the imaginary part of an adjoint chiral superfield after dimensional reduction as follows. Since the 5D $N=1$ SUSY theory corresponds to 4D $N=2$ SUSY theory, the 5D gauge multiplet,

$$
\mathcal{V}=\left(A^{M}, \lambda, \lambda^{\prime}, \sigma\right),
$$

is decomposed to a vector superfield and an adjoint chiral superfield as

$$
V=\left(A^{\mu}, \lambda\right), \quad \Sigma=\left(\sigma+i A^{5}, \lambda^{\prime}\right)
$$

respectively. Then, in the SUSY case, the gauge multiplet transforms as

$$
\left(\begin{array}{c}
V\left(x^{\mu},-y\right) \\
\Sigma\left(x^{\mu},-y\right)
\end{array}\right)=P\left(\begin{array}{c}
V\left(x^{\mu}, y\right) \\
-\Sigma\left(x^{\mu}, y\right)
\end{array}\right) P^{\dagger}
$$

$$
\left(\begin{array}{c}
V\left(x^{\mu}, \pi R-y\right) \\
\Sigma\left(x^{\mu}, \pi R-y\right)
\end{array}\right)=P^{\prime}\left(\begin{array}{c}
V\left(x^{\mu}, \pi R+y\right) \\
-\Sigma\left(x^{\mu}, \pi R+y\right)
\end{array}\right) P^{\prime \dagger},
$$

corresponding to Eqs. (1)-(4).

\section{III. $S U(3)_{c} \times S U(3)_{W}$ MODEL}

Let us study the possibility of the dynamical symmetry breaking in the $S U(3)_{C} \times S U(3)_{W}$ model, where the Higgs doublets can be identified as the zero mode components of $A_{5}[9-11]$. We take

$$
P=P^{\prime}=\operatorname{diag}(1,-1,-1)
$$

in the base of $S U(3)_{W} \cdot{ }^{2}$ Then, they divide $A_{\mu}$ and $A_{5}$ as

$$
\begin{gathered}
A_{\mu}=\left(\begin{array}{lll}
(+,+) & (-,-) & (-,-) \\
(-,-) & (+,+) & (+,+) \\
(-,-) & (+,+) & (+,+)
\end{array}\right), \\
A_{5}=\left(\begin{array}{lll}
(-,-) & (+,+) & (+,+) \\
(+,+) & (-,-) & (-,-) \\
(+,+) & (-,-) & (-,-)
\end{array}\right),
\end{gathered}
$$

which suggest $S U(3)_{W}$ is broken down to $S U(2)_{L}$ $\times U(1)_{Y}$, and there appear one "Higgs doublet" in $A_{5}$ as the zero mode. ${ }^{3}$ We assume that the compactification scale, $R^{-1}$, as a few $\mathrm{TeV}$.

The VEV of $A_{5}$ is written as

$$
\left\langle A_{5}\right\rangle=\frac{1}{g R} \sum_{a} a_{a} \frac{\lambda_{a}}{2},
$$

and we can always take VEV as

\footnotetext{
${ }^{2}$ As for $S U(3)_{c}$, we take $P=P^{\prime}=I$.

${ }^{3}$ In SUSY case, there appear two "Higgs doublets" as the zero modes.
} 


$$
a_{1}=a,
$$

and $a_{i}=0$ for $i \neq 1$ by using the residual $S U(2) \times U(1)$ global symmetry. The effective potential of $A_{5}$ is given by [9]

$$
V_{\mathrm{eff}}^{\mathrm{gauge}}=-\frac{3}{2} C \sum_{n=1}^{\infty} \frac{1}{n^{5}}[\cos (2 \pi n a)+2 \cos (\pi n a)],
$$

where $C \equiv 3 /\left(64 \pi^{7} R^{5}\right)$. This means that the point at $a=0$ is the minimum in $V_{\text {eff }}^{\text {gauge }}$, which suggests $S U(2)_{L} \times U(1)_{Y}$ is not broken.

Then, in order to realize the electroweak symmetry breaking, let us introduce extra fields in the bulk, which are $N_{s}$ numbers of complex scalars, $\phi$, and $N_{f}\left(N_{a}\right)$ numbers of Dirac fermions, $\psi\left(\psi^{a}\right)$, of the fundamental (adjoint) representation. They transform

$$
\begin{gathered}
\phi(x,-y)=\eta P \phi(x, y), \\
\phi(x, \pi R-y)=\eta^{\prime} P^{\prime} \phi(x, \pi R+y), \\
\psi(x,-y)=\eta P \gamma^{5} \psi(x, y), \\
\psi(x, \pi R-y)=\eta^{\prime} P^{\prime} \gamma^{5} \psi(x, \pi R+y), \\
\psi^{a}(x,-y)=\eta P \gamma^{5} \psi^{a}(x, y) P^{\dagger}, \\
\psi^{a}(x, \pi R-y)=\eta^{\prime} P^{\prime} \gamma^{5} \psi^{a}(x, \pi R+y) P^{\prime \dagger},
\end{gathered}
$$

under parities, respectively. Here $\eta, \eta^{\prime}= \pm$, and the effective potential induced from these bulk fields strongly depends on the sign of the product, $\eta \eta^{\prime}$. Appendix B shows the bulk fields' contributions to the effective potential,

$$
\begin{aligned}
V_{\mathrm{eff}}^{m}= & C \sum_{n=1}^{\infty} \frac{1}{n^{5}}\left[2 N_{a}^{(+)} \cos (2 \pi n a)\right. \\
& +2 N_{a}^{(-)} \cos \left(2 \pi n\left(a-\frac{1}{2}\right)\right) \\
& +\left(4 N_{a}^{(+)}-N_{s}^{(+)}+2 N_{f}^{(+)}\right) \cos (\pi n a) \\
& \left.+\left(4 N_{a}^{(-)}-N_{s}^{(-)}+2 N_{f}^{(-)}\right) \cos (\pi n(a-1))\right] .
\end{aligned}
$$

The index $( \pm)$ indicates the sign of $\eta \eta^{\prime}$ in Eqs. (16)-(18). Here we denote $N_{s}=N_{s}^{(+)}+N_{s}^{(-)}, N_{f}=N_{f}^{(+)}+N_{f}^{(-)}$, and $N_{a}=N_{a}^{(+)}+N_{a}^{(-)}$. Seeing the first derivative of $V_{\mathrm{eff}}=V_{\mathrm{eff}}^{\text {gauge }}$ $+V_{\text {eff }}^{m}$, each term of $\partial V_{\text {eff }} / \partial a$ has a factor $\sin (\pi n a)$, which means that the stationary points exist at least at $a=0$ and $a$ $=1{ }^{4}$ The difference of the heights between two points is given by

$$
\begin{aligned}
V_{\mathrm{eff}}(a= & 0)-V_{\mathrm{eff}}(a=1) \\
= & 2\left[4\left(N_{a}^{(+)}-N_{a}^{(-)}\right)\right. \\
& +2\left(N_{f}^{(+)}-N_{f}^{(-)}\right) \\
& \left.-\left(N_{s}^{(+)}-N_{s}^{(-)}\right)-3\right] C \sum_{n=1}^{\infty} \frac{1}{(2 n-1)^{5}} .
\end{aligned}
$$

This means that the symmetric point of $a=0$ becomes deeper as the number of bulk scalars with $\eta \eta^{\prime}=+$ and bulk fermions with $\eta \eta^{\prime}=-$ increase. On the other hand, the effects of scalars with $\eta \eta^{\prime}=-$ and fermions with $\eta \eta^{\prime}$ $=+$ make the height of $a=1$ decrease. When $a=1$ point becomes the vacuum, the Wilson loop becomes

$$
\begin{aligned}
W_{C} & =\exp \left(i g \int_{0}^{2 \pi R} d y \frac{1}{g R} a \frac{\lambda_{1}}{2}\right)=\exp \left(i g \frac{1}{g R} \frac{\lambda_{1}}{2} 2 \pi R\right) \\
& =\left(\begin{array}{ccc}
-1 & \\
& -1 & \\
& & 1
\end{array}\right)
\end{aligned}
$$

which suggests $S U(2)_{L} \times U(1)_{Y}$ is broken down to $U(1)_{e m}$ $\times U(1)$. Since the VEV is $\mathcal{O}\left(R^{-1}\right)$, which is a few TeV, this case cannot reproduce the correct weak scale VEV.

In order to realize the suitable electroweak symmetry breaking, we must find another vacuum at $(0<) a \ll 1$. In this case $S U(2)_{L} \times U(1)_{Y}$ is broken down to $U(1)_{e m}$. Seeing $V_{\text {eff }}=V_{\text {eff }}^{\text {gauge }}+V_{\text {eff }}^{m}$ in Eqs. (15) and (19), we notice that $n$ $=1$ (of the summation of $n$ ) has dominant contributions for the form of the effective potential. Thus, we can obtain the suitable value of $a(a \ll 1)$, by introducing bulk fields which induce large coefficients of $-\cos (\pi n a)$ and/or $\cos (\pi n(a$ $-1)$ ), and small (but nonzero) coefficients of $\cos (2 \pi n a)$ and $-\cos (2 \pi n(a-1 / 2))$. We show an example which satisfies the above condition, that is, $N_{a}^{(+)}=2, N_{f}^{(-)}=8, N_{s}^{(+)}=4$, $N_{s}^{(-)}=2$, and $N_{a}^{(-)}=N_{f}^{(+)}=0$. Figure 1 shows the $V_{\text {eff }}$ in the region of $0 \leqslant a \leqslant 1$ and $0 \leqslant a \leqslant 0.1$. The minimum exists at $a=0.058$, which is around the suitable magnitude of the weak scale in $\mathrm{TeV}$ scale compactification. To be more precise, the kinetic term of the "Higgs field" is obtained from the $5 \mathrm{D}$ gauge kinetic term,

\footnotetext{
${ }^{4}$ The potential has the symmetry $V_{\text {eff }}(-a)=V_{\text {eff }}(a)$, so that we should only check the region of $0 \leqslant a \leqslant 1$.
} 

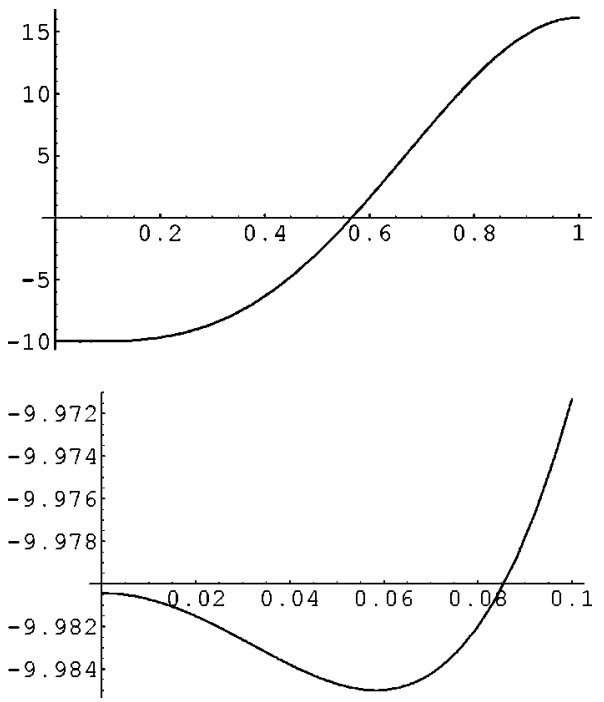

FIG. 1. The effective potential in the case of $N_{a}^{(+)}=2, N_{f}^{(-)}$ $=8, \quad N_{s}^{(+)}=4, \quad N_{s}^{(-)}=2$, and $N_{a}^{(-)}=N_{f}^{(+)}=0$. The unit is $C$ $=3 / 64 \pi^{7} R^{5}$. The horizontal line shows $0 \leqslant a \leqslant 1$ and $0 \leqslant a \leqslant 0.1$.

$$
\begin{aligned}
2 \times \int d y \frac{1}{4} F_{\mu 5}^{a} F^{a \mu 5} & =\int d y \frac{1}{2}\left(\partial_{\mu} A_{5}^{a}+i g f_{b c}^{a} A_{\mu}^{b} A_{5}^{c}\right)^{2} \\
& =\left|\left(\partial_{\mu}+i g_{4} W_{\mu}^{\alpha} \frac{\tau^{\alpha}}{2}+i \sqrt{3} g_{4} \frac{B_{\mu}}{2}\right) H\right|^{2} .
\end{aligned}
$$

Where $H=\sqrt{2 \pi R}\left(\left(A_{5}^{1}+i A_{5}^{2}\right) / \sqrt{2},\left(A_{5}^{4}+i A_{5}^{5}\right) / \sqrt{2}\right)^{T}$ is the 4D "Higgs doublet," and $g_{4}$ is the 4D gauge coupling constant defined as $g_{4}=g / \sqrt{2 \pi R}$. This yields too large $\sin \theta_{W}$ and it seems hard to reconcile it with the experimental value through the renormalization group effect. However, as discussed in Ref. [11], 4D gauge couplings can be also affected by wall-localized gauge kinetic terms, such as $\delta(0) \lambda_{0} F^{\mu \nu 2}$, which do not respect the bulk symmetry. When these couplings dominate bulk gauge couplings, ${ }^{5}$ we can expect to have the suitable gauge couplings in the low energy. In this case, the normalization of the low energy gauge fields might be changed as $\left(W_{\mu}, B_{\mu}\right) \rightarrow\left(g_{2} / g_{4} W_{\mu}, g_{Y} /\left(\sqrt{3} g_{4}\right) B_{\mu}\right)$, which yields the usual Higgs kinetic term. Thus, we set

$$
\sqrt{2 \pi R}\left\langle A_{5}^{1}\right\rangle=\frac{a}{g_{4} R} \sim 246 \mathrm{GeV} .
$$

The mass squared of the "Higgs field" is given by

\footnotetext{
${ }^{5}$ For this situation, we need two assumptions. One is that the wall-localized Higgs kinetic term is negligibly small compared to the bulk Higgs kinetic term. The other is that the bulk induced gauge coupling should be larger than wall-localized "gauge coupling" as, $g_{4}^{2}>\lambda_{0}^{-1}$. Thus, we should take $g_{4} \geqslant 1$, since the walllocalized "gauge couplings," such as $\lambda_{0}$, can mainly reproduce the magnitudes of low energy gauge couplings of $S U(2)_{L} \times U(1)_{Y}$, $\left(g_{2}, g_{Y}\right)$, under this situation. And we simply assume $g_{4}=\mathcal{O}(1)$ in the following discussions.
}

$$
\begin{aligned}
m_{A_{5}}^{2} & =\left.(g R)^{2} \frac{\partial^{2} V_{\mathrm{eff}}}{\partial a^{2}}\right|_{a=0.058} \\
& =\left.\frac{3 g_{4}^{2}}{32 \pi^{4} R^{2}} \frac{\partial^{2}\left(V_{\mathrm{eff}} /\left(C \pi^{2}\right)\right)}{\partial a^{2}}\right|_{a=0.058} .
\end{aligned}
$$

By using the approximation formula,

$$
\begin{gathered}
\sum_{n=1}^{\infty} \frac{\cos (n \pi \xi)}{n^{3}} \simeq \zeta(3)+\frac{(\pi \xi)^{2}}{2} \ln (\pi \xi)-\frac{3}{4}(\pi \xi)^{2}, \\
\sum_{n=1}^{\infty} \frac{\cos (n \pi(\xi-1))}{n^{3}} \simeq-\frac{3}{4} \zeta(3)+\frac{(\pi \xi)^{2}}{2} \ln 2,
\end{gathered}
$$

for a small $\xi$, the "Higgs" mass becomes

$$
m_{A_{6}}^{2} \sim\left(\frac{0.031 g_{4}}{R}\right)^{2} \sim\left(130 g_{4}^{2} \mathrm{GeV}\right)^{2}
$$

where $g_{4}=\mathcal{O}(1)$.

Now let us consider the SUSY case. Since the effective potential is zero when SUSY remains, we adopt ScherkSchwarz (SS) SUSY breaking [19-22], ${ }^{6}$ where the mode expansions are given in Ref. [4]. The effective potential in the SUSY version is given by

$$
\begin{aligned}
V_{\mathrm{eff}}^{\mathrm{gauge}}= & -2 C \sum_{n=1}^{\infty} \frac{1}{n^{5}}(1-\cos (2 \pi n \beta))[\cos (2 \pi n a) \\
& +2 \cos (\pi n a)]
\end{aligned}
$$

where $\beta$ parametrizes SS SUSY breaking. We take $\beta \sim 0.1$, since the soft mass is given by $\beta / R$ [4]. Since (1 $-\cos (2 \pi n \beta)) \geqslant 0$, Eq. (26) means that $a=0$ is the minimum point in the effective potential. Therefore, the $S U(2)_{L}$ $\times U(1)_{Y}$ is not broken as in the non-SUSY case.

What is going on if the extra fields exist in the bulk? We take the bulk fields of $N_{f}$ and $N_{a}$ species of hypermultiplets of fundamental $(\Psi)$ and adjoint $\left(\Psi^{a}\right)$ representations, respectively. The bulk hypermultiplets, $\Psi=\left(\phi, \phi^{c \dagger}, \widetilde{\phi}, \widetilde{\phi}^{c \dagger}\right)$ and $\Psi^{a}=\left(\phi^{a}, \phi^{a c \dagger}, \widetilde{\phi}^{a}, \widetilde{\phi}^{a c \dagger}\right)$, which are decomposed into chiral superfields as, $\Phi=(\phi, \widetilde{\phi}), \Phi^{a}=\left(\phi^{a}, \widetilde{\phi}^{a}\right)$, and $\Phi^{c}$ $=\left(\phi^{c}, \widetilde{\phi}^{c}\right), \Phi^{a c}=\left(\phi^{a c}, \widetilde{\phi}^{a c}\right)$, where $\Phi\left(\Phi^{a}\right)$ and $\Phi^{c}\left(\Phi^{a c}\right)$ have conjugated transformation under the gauge group. They transform

\footnotetext{
${ }^{6}$ For the effective potential in other SUSY breaking, the calculation has been done in the case of the $S^{1}$ compactification in Ref. [23].
} 


$$
\begin{aligned}
& \left(\begin{array}{c}
\Phi\left(x^{\mu},-y\right) \\
\Phi^{c^{\dagger}}\left(x^{\mu},-y\right)
\end{array}\right)=\eta P\left(\begin{array}{c}
\Phi\left(x^{\mu}, y\right) \\
-\Phi^{c \dagger}\left(x^{\mu}, y\right)
\end{array}\right) \\
& \left(\begin{array}{c}
\Phi\left(x^{\mu}, \pi R-y\right) \\
\Phi^{c^{\dagger}}\left(x^{\mu}, \pi R-y\right)
\end{array}\right)=\eta^{\prime} P^{\prime}\left(\begin{array}{c}
\Phi\left(x^{\mu}, \pi R+y\right) \\
-\Phi^{c \dagger}\left(x^{\mu}, \pi R+y\right)
\end{array}\right) \\
& \left(\begin{array}{c}
\Phi^{a}\left(x^{\mu},-y\right) \\
\Phi^{a c \dagger}\left(x^{\mu},-y\right)
\end{array}\right)=\eta P\left(\begin{array}{c}
\Phi^{a}\left(x^{\mu}, y\right) \\
-\Phi^{a c \dagger}\left(x^{\mu}, y\right)
\end{array}\right) P^{\dagger}, \\
& \left(\begin{array}{c}
\Phi^{a}\left(x^{\mu}, \pi R-y\right) \\
\Phi^{a c \dagger}\left(x^{\mu}, \pi R-y\right)
\end{array}\right)=\eta^{\prime} P^{\prime}\left(\begin{array}{c}
\Phi^{a}\left(x^{\mu}, \pi R+y\right) \\
-\Phi^{a c \dagger}\left(x^{\mu}, \pi R+y\right)
\end{array}\right) P^{\prime \dagger},
\end{aligned}
$$

under the parities, respectively. According to the sign of $\eta \eta^{\prime}$ we denote $N_{f}=N_{f}^{(+)}+N_{f}^{(-)}$and $N_{a}=N_{a}^{(+)}+N_{a}^{(-)}$. We always take even number of $N_{f}^{( \pm)}$to avoid the gauge anomaly. Appendix B suggests that the extra matter contributions for the effective potential are given by

$$
\begin{aligned}
V_{\mathrm{eff}}^{m}= & 2 C \sum_{n=1}^{\infty} \frac{1}{n^{5}}(1-\cos (2 \pi n \beta)) \\
& \times\left[N_{a}^{(+)} \cos (2 \pi n a)+N_{a}^{(-)} \cos \left(2 \pi n\left(a-\frac{1}{2}\right)\right)\right. \\
& +2\left(N_{a}^{(+)}+\frac{N_{f}^{(+)}}{2}\right) \cos (\pi n a) \\
& \left.+2\left(N_{a}^{(-)}+\frac{N_{f}^{(-)}}{2}\right) \cos (\pi n(a-1))\right]
\end{aligned}
$$

As the non-SUSY case, the first derivative of $V_{\text {eff }}=V_{\text {eff }}^{\text {gauge }}$ $+V_{\text {eff }}^{m}$ has the factor $\sin (\pi n a)$, which means the stationary points exist at $a=0$ and $a=1$. The difference of the heights between two points is given by

$$
\begin{aligned}
V_{\mathrm{eff}}(a=0) & -V_{\mathrm{eff}}(a=1) \\
= & -8\left[1-\left(N_{a}^{(+)}-N_{a}^{(-)}\right)-\frac{\left(N_{f}^{(+)}-N_{f}^{(-)}\right)}{2}\right] \\
& \times C \sum_{n=1}^{\infty} \frac{1}{(2 n-1)^{5}}(1-\cos (2 \pi(2 n-1) \beta)) .
\end{aligned}
$$

This means that the height of the point at $a=0$ becomes high (low) as increasing the number of $N_{a}^{(+)}\left(N_{a}^{(-)}\right)$and $N_{f}^{(+)}$ $\left(N_{f}^{(-)}\right)$. Equation (32) is consistent with the results in Ref. [5]. As for the case of $N_{a}^{(+)}=1, N_{f}^{(+)}=N_{f}^{(-)}=N_{a}^{(-)}=0$, the effective potential vanishes as $V_{\text {eff }} \equiv 0$, since this case has $5 \mathrm{D}$ $N=2$ SUSY and there is the residual SUSY after the SS SUSY breaking [24].
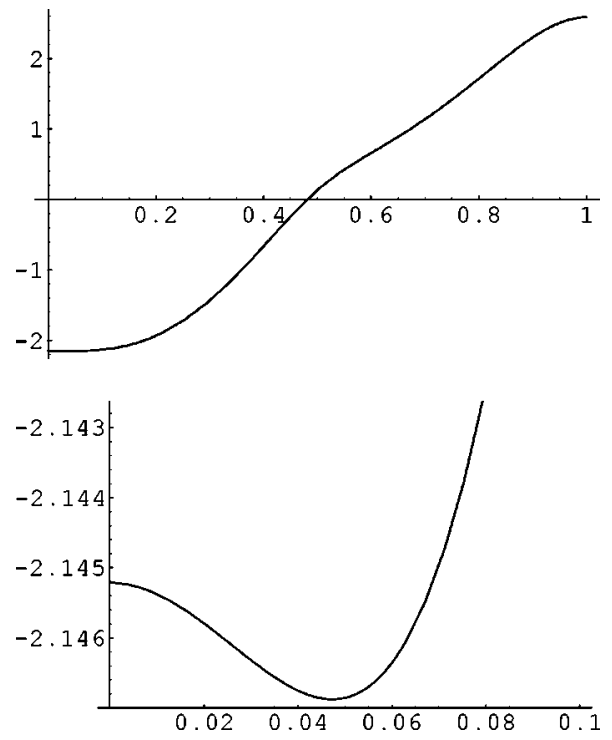

FIG. 2. The effective potential in the case of $N_{a}^{(+)}=N_{a}^{(-)}=2$, $N_{f}^{(-)}=4, N_{f}^{(+)}=0$ with $\beta=0.1$. The unit is $C=3 / 64 \pi^{7} R^{5}$. The horizontal line shows $0 \leqslant a \leqslant 1$ and $0 \leqslant a \leqslant 0.1$.

As the non-SUSY case, in order to obtain the suitable value of $a(a \ll 1)$, we should introduce bulk hypermultiplets which induce large coefficients of $-\cos (\pi n a)$ and/or $\cos (\pi n(a-1))$ and small (but nonzero) coefficients of $\cos (2 \pi n a)$ and $-\cos (2 \pi n(a-1 / 2))$. We show an example which satisfies the above condition, that is, $N_{a}^{(+)}=N_{a}^{(-)}=2$, $N_{f}^{(-)}=4, N_{f}^{(+)}=0$ (Fig. 2). In this case with $\beta=0.1$, the minimum exists at $a=0.047$, and the "Higgs" mass squared is given by

$$
m_{A_{5}}^{2} \sim\left(\frac{0.025 g_{4}}{R}\right)^{2} \sim\left(130 g_{4}^{2} \mathrm{GeV}\right)^{2}
$$

where $g_{4}=\mathcal{O}(1)$. It should be noticed that the numerical analysis shows that the value of $a$ depends on that of $\beta$.

\section{IV. $S U(6)$ MODEL}

We study the vacuum structure of the $S U(6)$ GUT, in which the Higgs doublets can be identified as the zero mode components of $A_{5}[10,11]$. We take

$$
\begin{gathered}
P=\operatorname{diag}(1,1,1,1,-1,-1), \\
P^{\prime}=\operatorname{diag}(1,-1,-1,-1,-1,-1),
\end{gathered}
$$

which divide $A_{\mu}$ and $A_{5}$ as 


$$
\begin{gathered}
A_{\mu}=\left(\begin{array}{llllll}
(+,+) & (+,-) & (+,-) & (+,-) & (-,-) & (-,-) \\
(+,-) & (+,+) & (+,+) & (+,+) & (-,+) & (-,+) \\
(+,-) & (+,+) & (+,+) & (+,+) & (-,+) & (-,+) \\
(+,-) & (+,+) & (+,+) & (+,+) & (-,+) & (-,+) \\
(-,-) & (-,+) & (-,+) & (-,+) & (+,+) & (+,+) \\
(-,-) & (-,+) & (-,+) & (-,+) & (+,+) & (+,+)
\end{array}\right), \\
\left.A_{5}=\mid \begin{array}{llllll}
(-,-) & (-,+) & (-,+) & (-,+) & (+,+) & (+,+) \\
(-,+) & (-,-) & (-,-) & (-,-) & (+,-) & (+,-) \\
(-,+) & (-,-) & (-,-) & (-,-) & (+,-) & (+,-) \\
(-,+) & (-,-) & (-,-) & (-,-) & (+,-) & (+,-) \\
(+,+) & (+,-) & (+,-) & (+,-) & (-,-) & (-,-) \\
(+,+) & (+,-) & (+,-) & (+,-) & (-,-) & (-,-)
\end{array}\right) .
\end{gathered}
$$

They suggest that $P$ and $P^{\prime}$ make $S U(6)$ broken to $S U(3)_{c} \times S U(2)_{L} \times U(1)_{Y} \times U(1)$. Also, there appears to be one "Higgs doublet" in $A_{5}$ as the zero mode. As in the preceding section, the compactification scale is assumed to be of order a few TeV. The VEV of $A_{5}$ is written in the similar way as Eq. (14) as

$$
\left\langle A_{5}\right\rangle=\frac{1}{g R} a \frac{\lambda_{16}}{2}
$$

by using the residual $S U(2) \times U(1)$ global symmetry. The calculation in Appendix A suggests the gauge part of the effective potential in the $S U(6)$ model as

$$
\begin{aligned}
V_{\mathrm{eff}}^{\text {gauge }}= & -\frac{3}{2} C \sum_{n=1}^{\infty} \frac{1}{n^{5}}[6 \cos (\pi n(a-1)) \\
& +2 \cos (\pi n a)+\cos (2 \pi n a)] .
\end{aligned}
$$

From this equation, we can easily show that

$$
\begin{aligned}
V_{\mathrm{eff}}^{\text {gauge }}(a=0)-V_{\mathrm{eff}}^{\mathrm{gauge}}(a=1) \\
\quad=12 C \sum_{n=1}^{\infty} \frac{1}{(2 n-1)^{5}}>0 .
\end{aligned}
$$

The numerical calculation of the effective potential shows us that $a=1(a=0)$ point is the global (local) minimum. The vacuum at $a=1$ has the Wilson loop

$$
\begin{aligned}
W_{C} & =\exp \left(i g \int_{0}^{2 \pi R} d y \frac{1}{g R} a \frac{\lambda_{16}}{2}\right) \\
& =\exp \left(i g \frac{1}{g R} \frac{\lambda_{16}}{2} 2 \pi R\right) \\
& =\left(\begin{array}{ccccc}
-1 & & & \\
& 1 & & & \\
& & 1 & & \\
& & 1 & & \\
& & & -1 & \\
& & & & 1
\end{array}\right),
\end{aligned}
$$

which suggests that $S U(2)_{L} \times U(1)_{Y}$ is broken down to $U(1)_{e m} \times U(1)$. This means that the vacuum at $a=1$ is not suitable, since the weak scale becomes too large. It can be possible to assume that we exist at $a=0$ in the early universe, and the lifetime of this false vacuum is longer than the universe history [4]. However, the suitable electroweak symmetry breaking cannot be realized, anyway.

Then, let us introduce the extra fields in the bulk for the suitable dynamical symmetry breaking of $S U(2)_{L} \times U(1)_{Y}$. We introduce the bulk fields of $N_{s}$ numbers of complex scalars, $\phi$, and $N_{f}\left(N_{a}\right)$ numbers of Dirac fermions, $\psi\left(\psi^{a}\right)$, of the fundamental (adjoint) representation. The transformations under parities are the same as Eqs. (16)-(18). Appendix B suggests that the effective potential induced from the bulk fields is given by 


$$
\begin{aligned}
V_{\mathrm{eff}}^{m}= & C \sum_{n=1}^{\infty} \frac{1}{n^{5}}\left[2 N_{a}^{(+)} \cos (2 \pi n a)\right. \\
& +2 N_{a}^{(-)} \cos \left(2 \pi n\left(a-\frac{1}{2}\right)\right) \\
& +\left(4 N_{a}^{(+)}+12 N_{a}^{(-)}+2 N_{f}^{(+)}-N_{s}^{(+)}\right) \cos (\pi n a) \\
& +\left(12 N_{a}^{(+)}+4 N_{a}^{(-)}+2 N_{f}^{(-)}\right. \\
& \left.\left.-N_{s}^{(-)}\right) \cos (\pi n(a-1))\right]
\end{aligned}
$$

The index $( \pm)$ shows the numbers of bulk fields with the sign of $\eta \eta^{\prime}$ in Eqs. (16)-(18). We denote as $N_{s}=N_{s}^{(+)}$ $+N_{s}^{(-)}, N_{f}=N_{f}^{(+)}+N_{f}^{(-)}$, and $N_{a}=N_{a}^{(+)}+N_{a}^{(-)}$. As in the preceding section, the first derivative of $V_{\text {eff }}=V_{\mathrm{eff}}^{\text {gauge }}+V_{\mathrm{eff}}^{m}$ suggests that the stationary points exist at $a=0$ and $a=1$. The difference of the heights between two points is given by

$$
\begin{aligned}
V^{\mathrm{eff}}(a= & 0)-V^{\mathrm{eff}}(a=1) \\
= & 2\left[6-8\left(N_{a}^{(+)}-N_{a}^{(-)}\right)\right. \\
& +2\left(N_{f}^{(+)}-N_{f}^{(-)}\right) \\
& \left.-\left(N_{s}^{(+)}-N_{s}^{(-)}\right)\right] C \sum_{n=1}^{\infty} \frac{1}{(2 n-1)^{5}} .
\end{aligned}
$$

The symmetric point of $a=0$ becomes deeper (higher) as $N_{a}^{(+)}, N_{f}^{(-)}, N_{s}^{(+)}\left(N_{a}^{(-)}, N_{f}^{(+)}, N_{s}^{(-)}\right)$increase.

As in the preceding section, in order to obtain the suitable value of $a(a \ll 1)$, we should introduce bulk fields which induce large coefficients of $-\cos (\pi n a)$ and/or $\cos (\pi n(a$ $-1)$ ) and small (but nonzero) coefficients of $\cos (2 \pi n a)$ and $-\cos (2 \pi n(a-1 / 2))$. We show an example which satisfies the above condition, that is, $N_{a}^{(+)}=N_{f}^{(-)}=2$, and others being zero (Fig. 3). In this case, the minimum exists at $a$ $=0.072$, and the "Higgs" mass squared is given by

$$
m_{A_{5}}^{2} \sim\left(\frac{0.038 g_{4}}{R}\right)^{2} \sim\left(130 g_{4}^{2} \mathrm{GeV}\right)^{2}
$$

where $g_{4}=\mathcal{O}(1)$.

The effective potential in the SUSY case is given by

$$
\begin{aligned}
V_{\mathrm{eff}}^{\mathrm{gauge}}= & -2 C \sum_{n=1}^{\infty} \frac{1}{n^{5}}(1-\cos (2 \pi n \beta)) \\
& \times[6 \cos (\pi n(a-1))+2 \cos (\pi n a) \\
& +\cos (2 \pi n a)]
\end{aligned}
$$

from the calculation of Appendix A. This shows
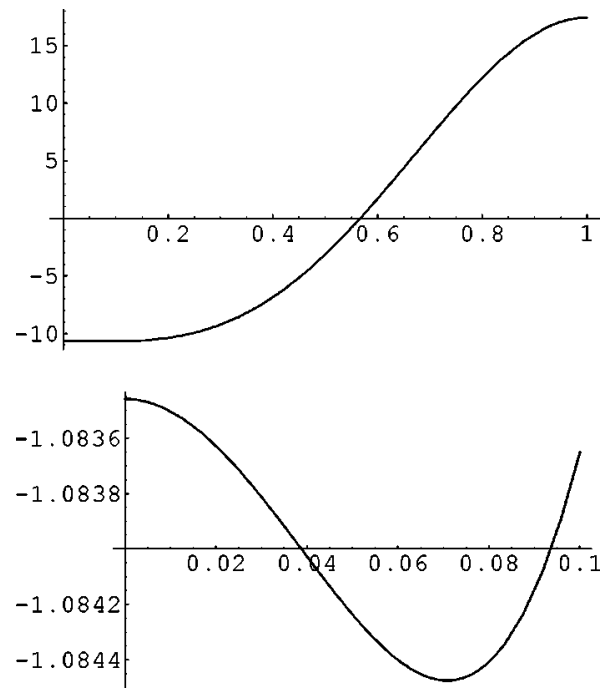

FIG. 3. The effective potential in the case of $N_{a}^{(+)}=N_{f}^{(-)}=2$, and others being zero. The unit is $C=3 / 64 \pi^{7} R^{5}$. The horizontal line shows $0 \leqslant a \leqslant 1$ and $0 \leqslant a \leqslant 0.1$.

$$
\begin{aligned}
V_{\mathrm{eff}}^{\mathrm{gauge}}(a= & 0)-V_{\mathrm{eff}}^{\mathrm{gauge}}(a=1) \\
= & 16 C \sum_{n=1}^{\infty} \frac{1}{(2 n-1)^{5}} \\
& \times(1-\cos (2 \pi(2 n-1) \beta))>0,
\end{aligned}
$$

and the numerical calculation really shows $a=1$ is the global minimum. Then, in order to realize the suitable electroweak symmetry breaking, we introduce the extra hypermultiplets in the bulk as in the preceding section. We introduce $N_{f}\left(N_{a}\right)$ numbers of fundamental (adjoint) hypermultiplets, which transform Eqs. (27) and (28) [Eqs. (29) and (30)] under parities with $\eta \eta^{\prime}= \pm$. Appendix B shows that the bulk fields induce the effective potential,

$$
\begin{aligned}
V_{\mathrm{eff}}^{m}= & 2 C \sum_{n=1}^{\infty} \frac{1}{n^{5}}(1-\cos (2 \pi n \beta))\left[N_{a}^{(+)} \cos (2 \pi n a)\right. \\
& +N_{a}^{(-)} \cos \left(2 \pi n\left(a-\frac{1}{2}\right)\right) \\
& +\left(2 N_{a}^{(+)}+6 N_{a}^{(-)}+N_{f}^{(+)}\right) \cos (\pi n a) \\
& \left.+\left(6 N_{a}^{(+)}+2 N_{a}^{(-)}+N_{f}^{(-)}\right) \cos (\pi n(a-1))\right]
\end{aligned}
$$

We can show that the effective potential vanishes in the case of $N_{a}^{(+)}=1, N_{f}^{(+)}=N_{f}^{(-)}=N_{a}^{(-)}=0$ due to the residual SUSY [24]. The first derivative of $V_{\text {eff }}=V_{\mathrm{eff}}^{\mathrm{gauge}}+V_{\mathrm{eff}}^{m}$ suggests that the stationary points exist at $a=0$ and $a=1$. The difference of the heights between two points is given by 

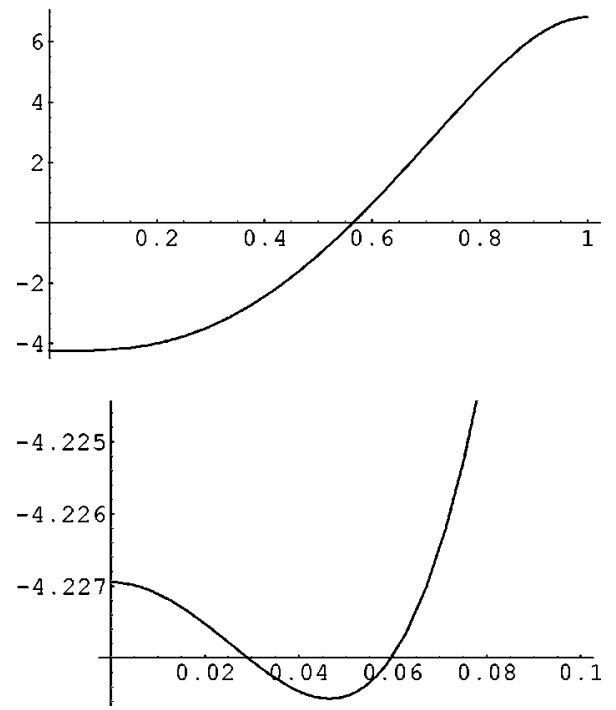

FIG. 4. The effective potential in the case of $N_{a}^{(+)}=2, N_{f}^{(-)}$ $=10, N_{a}^{(-)}=N_{f}^{(+)}=0$ with $\beta=0.1$. The unit is $C=3 / 64 \pi^{7} R^{5}$. The horizontal line shows $0 \leqslant a \leqslant 1$ and $0 \leqslant a \leqslant 0.1$.

$$
\begin{aligned}
V_{\mathrm{eff}}(0)-V_{\mathrm{eff}}(1)= & 2\left(8-8\left(N_{a}^{(+)}-N_{a}^{(-)}\right)\right. \\
& \left.+2\left(N_{f}^{(+)}-N_{f}^{(-)}\right)\right) C \sum_{n=1}^{\infty} \frac{1}{(2 n-1)^{5}} \\
& \times(1-\cos (2 \pi(2 n-1) \beta)),
\end{aligned}
$$

which is consistent with the results in Ref. [5].

As the non-SUSY case, in order to obtain the suitable value of $a(a \ll 1)$, we should introduce bulk hypermultiplets which induce large coefficients of $-\cos (\pi n a)$ and/or $\cos (\pi n(a-1))$ and small (but nonzero) coefficients of $\cos (2 \pi n a)$ and $-\cos (2 \pi n(a-1 / 2))$. We show an example that satisfies the above condition, that is, $N_{a}^{(+)}=2, N_{f}^{(-)}$ $=10, N_{a}^{(-)}=N_{f}^{(+)}=0$ with $\beta=0.1$ (Fig. 4). In this case, the minimum exists at $a=0.047$, and the "Higgs" mass squared is given by

$$
m_{A_{5}}^{2} \sim\left(\frac{0.024 g_{4}}{R}\right)^{2} \sim\left(130 g_{4}^{2} \mathrm{GeV}\right)^{2},
$$

where $g_{4}=\mathcal{O}(1)$.

As for the extra $U(1)$ gauge symmetry that remains unbroken through the orbifolding boundary conditions, we assume it is broken by an extra elementally Higgs field.

\section{SUMMARY AND DISCUSSION}

We have studied the possibility of the dynamical symmetry breaking in the gauge-Higgs unification in the 5D theory compactified on an orbifold, $S^{1} / Z_{2}$. This theory identifies Wilson line degrees of freedom as "Higgs doublets." We considered $S U(3)_{c} \times S U(3)_{W}$ and $S U(6)$ models with the compactification scale of order of a few $\mathrm{TeV}$. The gauge symmetries are reduced to $S U(3)_{c} \times S U(2)_{L} \times U(1)_{Y}$ and $S U(3)_{c} \times S U(2)_{L} \times U(1)_{Y} \times U(1)$, respectively, through the orbifolding boundary conditions. We have estimated the one loop effective potential of "Higgs doublets," and find that the electroweak symmetry breaking is realized through the radiative corrections when there are suitable numbers of bulk fields possessing the suitable representations. The masses of "Higgs doublets" are $\mathcal{O}(100) \mathrm{GeV}$ in this scenario.

Although the compactification scale is a few $\mathrm{TeV}$, the contributions from the higher dimensional operators to the one loop effective potential are suppressed enough in this model. It is because $g_{4}=\mathcal{O}(1)$ suggests that the cutoff scale $M$ can be larger than the compactification scale, $1 / R$, enough to satisfy $(R M) \lessgtr 4 \pi$, where gauge interaction is still perturbative as $\alpha=g_{4}^{2} / 4 \pi \lesssim 1$. Thus, there appears the suppression factor, $\mathcal{O}(1 / R M)$, for the higher dimensional operators in the effective potential. As for the contributions of them to the tree-level scalar potential, they vanish as long as fields other than Wilson line phases do not acquire VEVs. We analyze the vacuum on the direction of Wilson line phases, $\left\langle A_{5}\right\rangle$ in this paper.

Finally we should comment on the Yukawa interactions. We have assumed that quarks and leptons are localized on the 4D wall. In this situation, the "Higgs doublet" cannot make the gauge invariant Yukawa interactions even if the "Higgs doublet" appear as the zero mode of $\Sigma$ as is shown in Ref. [10]. It is because $\Sigma$ transforms as $\Sigma \rightarrow e^{\Lambda}(\Sigma$ $\left.-\sqrt{2} \partial_{y}\right) e^{-\Lambda}$ under the gauge transformation. However, if we consider the nonlocal operator, $\phi=\mathcal{P} \exp \left(\int \Sigma d y\right)$, as the new Higgs doublet, it can have the gauge invariant Yukawa interactions with the wall-localized quarks and leptons [10]. Another possibility is to consider the situation where quarks and leptons are the bulk fields [11], where the Yukawa interactions is originated in the 5D gauge interactions.

Note added in proof. After this work was completed we noticed the work of Scrucca, Serone, and Silvestrini [25], where a similar idea is considered in non-SUSY $S U(3)_{c}$ $\times S U(3)_{W}$. They estimated the effects to the effective potential induced from bulk and wall-localized fields mixings and wall-localized kinetic terms. They also consider flavor symmetry breaking through Yukawa interactions with the nonlocal operator induced by integrating out heavy bulk fields [26]. The difference between their scenario and ours is that the electroweak symmetry breaking is realized by the effect of wall-localized fields in the former, while by the effect of bulk fields possessing degrees of freedom of $\eta$ and $\eta^{\prime}$ in the latter.

\section{ACKNOWLEDGMENTS}

N.H. would like to thank C. S. Lim for a lot of very helpful discussions. T.Y. would like to thank S. Teraguchi for useful discussions, and is supported by a Grant-in-Aid for the 21st Century COEhCenter for Diversity and Universality in Physicsh. This work was supported in part by Scientific Grants from the Ministry of Education and Science, Grants Nos. 14039207, 14046208, 14740164 (N.H.), 13135215, 13640284 (Y.H.), 13135217, 15340078 (Y.K.).

\section{APPENDIX A: THE DERIVATION OF $V_{\mathrm{eff}}^{\text {gauge }}$ IN $S U(6)$}

The one loop effective potential of the Wilson line degrees of freedom of $A_{5}$ is given by 


$$
V_{\mathrm{eff}}\left[A^{0}\right]=-(D-2) \frac{i}{2} \operatorname{Tr} \ln D_{M}^{0} D^{0 M},
$$

where $D_{L}\left(A^{0}\right) D^{L}\left(A^{0}\right)=\partial_{\mu} \partial^{\mu}+D_{y}\left(A^{0}\right) D^{y}\left(A^{0}\right)$, with $A^{0}$ $\equiv\left\langle A_{5}\right\rangle=(1 / g R) \Sigma_{a} a_{a}\left(\lambda_{a} / 2\right)$. We can always take the base of $\left\langle A_{5}\right\rangle=0$ by the gauge transformation, $\Omega\left(x^{\mu}, y\right)$ $=e^{i \sum a_{a}\left(\lambda_{a} / 2\right)(y / R)}$, as

$$
\begin{aligned}
\left\langle A_{5}\right\rangle \rightarrow & \Omega(y)\left\langle A_{5}\right\rangle \Omega(y)^{\dagger}-\frac{i}{g} \Omega(y) \partial_{y} \Omega(y)^{\dagger}=\left\langle A_{5}\right\rangle \\
& -\frac{1}{g R} \sum a_{a} \frac{\lambda_{a}}{2}=0 .
\end{aligned}
$$

In this base the parities are given as

$$
\begin{aligned}
P \rightarrow P & =\Omega(-y) P \Omega(y)^{\dagger}, \\
P^{\prime} \rightarrow P^{\prime} & =\Omega(\pi R-y) P^{\prime} \Omega(\pi R+y)^{\dagger} .
\end{aligned}
$$

Then $P$ and $P^{\prime}$ correspond to the Wilson loop $W_{C}$.
Introducing a fluctuation field $B \equiv \Sigma_{a} B_{a}\left(\lambda_{a} / 2\right)$, the effective potential is given by

$\operatorname{Tr} \ln B D_{y}\left(A^{0}\right) D_{y}\left(A^{0}\right) B$

$$
=-\operatorname{Tr}\left(\sum_{c}\left[\partial_{y} B_{c}-\frac{1}{R} \sum_{a, b} f_{a b c} a_{a} B_{b}\right] \frac{\lambda_{c}}{2}\right)^{2} .
$$

As is shown in the Sec. III, we can always take VEV as $a_{16}=a$, and other $a_{i}=0$, by using the residual $S U(2)$ $\times U(1)$ global symmetry. Thus, only structure constants relating $\lambda_{16}$ are needed for the calculation of Eq. (A5). They are given by

$$
\begin{aligned}
f_{1,16,19} & =f_{2,16,18}=f_{3,16,17}=f_{4,16,21}=f_{5,16,20}=f_{9,16,23}=f_{10,16,22} \\
& =f_{16,25,34}=f_{16,33,26}=\frac{1}{2},
\end{aligned}
$$$$
f_{8,16,17}=\frac{1}{2 \sqrt{3}}, \quad f_{15,16,17}=\frac{1}{2 \sqrt{6}}, \quad f_{16,17,24}=\frac{\sqrt{10}}{4} \text {. }
$$

Where the generators are numbered as

$$
\left(\begin{array}{cccccc} 
& (1,2) & (4,5) & (9,10) & (16,17) & (25,26) \\
(1,2) & & (6,7) & (11,12) & (18,19) & (27,28) \\
(4,5) & (6,7) & & (13,14) & (20,21) & (29,30) \\
(9,10) & (11,12) & (13,14) & & (22,23) & (31,32) \\
(16,17) & (18,19) & (20,21) & (22,23) & & (33,34) \\
(25,26) & (27,28) & (29,30) & (31,32) & (33,34) &
\end{array}\right) .
$$

For examples, $(1,2)$ stands for

$$
\lambda_{1}=\left(\begin{array}{ll}
1 & 1 \\
1 & \\
&
\end{array}\right), \quad \lambda_{2}=\left(\begin{array}{l}
i^{-i} \\
\end{array}\right)
$$

The diagonal generators are

$$
\begin{aligned}
& \lambda_{3}=\operatorname{diag}(1,-1,0,0,0,0), \quad \lambda_{8}=\frac{1}{\sqrt{3}} \operatorname{diag}(1,1,-2,0,0,0), \\
& \lambda_{15}=\frac{1}{\sqrt{6}} \operatorname{diag}(1,1,1,-3,0,0), \quad \lambda_{24}=\frac{1}{\sqrt{10}} \operatorname{diag}(1,1,1,1,-4,0), \\
& \lambda_{35}=\frac{1}{\sqrt{15}} \operatorname{diag}(1,1,1,1,1,-5),
\end{aligned}
$$




$$
\begin{gathered}
\frac{1}{2}\left[\left(\partial_{y} B_{i}\right)^{2}+\left(\partial_{y} B_{j}-\frac{1}{2 R} a B_{k}\right)^{2}+\left(\partial_{y} B_{k}+\frac{1}{2 R} a B_{j}\right)^{2}+\left(\partial_{y} B_{3}-\frac{1}{2 R} a B_{17}\right)^{2}+\left(\partial_{y} B_{8}-\frac{1}{2 \sqrt{3} R} a B_{17}\right)^{2}+\left(\partial_{y} B_{15}\right.\right. \\
\left.\left.-\frac{1}{2 \sqrt{6} R} a B_{17}\right)^{2}+\left(\partial_{y} B_{24}-\frac{\sqrt{10}}{4 R} a B_{17}\right)^{2}+\left(\partial_{y} B_{17}+\frac{1}{2 R} a B_{3}+\frac{1}{2 \sqrt{3} R} a B_{8}+\frac{1}{2 \sqrt{6} R} a B_{15}+\frac{\sqrt{10}}{4 R} a B_{24}\right)^{2}\right],
\end{gathered}
$$

where $i=6,7,11,12,13,14,16,27,28,29,30,31,32,35$, and $(j, k)=(1,19),(2,18),(4,21),(5,20),(9,23),(10,22),(25$, 34), (26, 33). In $i=6,7,11,12,13,14,35$ in Eq. (A10), eigenvalues are $n^{2} / R^{2}$ and $(n+1)^{2} / R^{2}$ for $(+,+)$ states of $A_{\mu}$ and $(-,-)$ states of $A_{5}$, respectively. In $i=16$ in Eq. (A10), eigenvalue is $(n+1)^{2} / R^{2}$ and $n^{2} / R^{2}$ for $(-,-)$ state of $A_{\mu}$ and $(+$, + ) state of $A_{5}$, respectively. In $i=27,28,29,30,31,32$ in Eq. (A10), eigenvalues are $(n+1 / 2)^{2} / R^{2}$ and $(n+1 / 2)^{2} / R^{2}$ for $(-$, $+)$ states of $A_{\mu}$ and $(+,-)$ states of $A_{5}$, respectively. In $(j, k)=(1,19),(2,18),(4,21),(5,20),(9,23),(10,22)$, eigenvalues are $(n+1 / 2+a / 2)^{2} / R^{2},(n+1 / 2-a / 2)^{2} / R^{2}$ and $(n+1 / 2+a / 2)^{2} / R^{2},(n+1 / 2-a / 2)^{2} / R^{2}$ for $((+,-),(-,+))$ states of $A_{\mu}$ and $((-,+),(+,-))$ states of $A_{5}$, respectively. In $(j, k)=(25,34),(26,33)$, eigenvalues are $(n+a / 2)^{2} / R^{2},(n-a / 2)^{2} / R^{2}$ and $(n+a / 2)^{2} / R^{2},(n-a / 2)^{2} / R^{2}$ for $((-,-),(+,+))$ states of $A_{\mu}$ and $((+,+),(-,-))$ states of $A_{5}$, respectively. As for $\left(B_{3}, B_{8}, B_{15}, B_{24}, B_{17}\right)$, the relevant part in Eq. (A10) is simplified as

$$
\frac{1}{2}\left\{\left(\partial_{y} C_{1}-\frac{a}{R} B_{17}\right)^{2}+\left(\partial_{y} B_{17}+\frac{a}{R} C_{1}\right)^{2}+\left(\partial_{y} C_{2}\right)^{2}+\left(\partial_{y} C_{3}\right)^{2}+\left(\partial_{y} C_{4}\right)^{2}\right\}
$$

in an appropriate basis. Here new fields $C_{j}$ 's are introduced by

$$
C_{1} \Lambda_{1}+C_{2} \Lambda_{2}+C_{3} \Lambda_{3}+C_{4} \Lambda_{4}=B_{3} \lambda_{3}+B_{8} \lambda_{8}+B_{15} \lambda_{15}+B_{24} \lambda_{24}
$$

by the use of new bases $\Lambda_{j}$ 's with diagonal elements. For instance, $C_{1}$ and $\Lambda_{1}$ are given by

$$
C_{1}=\frac{1}{2} B_{3}+\frac{1}{2 \sqrt{3}} B_{8}+\frac{1}{2 \sqrt{6}} B_{15}+\frac{\sqrt{10}}{4} B_{24}
$$

and

$$
\Lambda_{1}=\operatorname{diag}(1,0,0,0,-1,0)
$$

respectively. From Eq. (A11), the eigenvalues are given by $(n+a)^{2} / R^{2},(n-a)^{2} / R^{2}, n^{2} / R^{2}, n^{2} / R^{2}$, and $n^{2} / R^{2}$.

Then, the effective potential for gauge and ghost is given by

$$
\begin{aligned}
V_{\mathrm{eff}}^{g+g h}= & -2 \frac{i}{2} \int \frac{d^{4} p}{(2 \pi)^{4}} \frac{1}{2 \pi R}\left[\sum_{i} \sum_{n=0}^{\infty} \ln \left(-p^{2}+\frac{n^{2}}{R^{2}}\right)+\sum_{j} \sum_{n=1}^{\infty} \ln \left(-p^{2}+\frac{n^{2}}{R^{2}}\right)+\sum_{k} \sum_{n=0}^{\infty} \ln \left(-p^{2}+\frac{(n+1 / 2)^{2}}{R^{2}}\right)\right. \\
& +\sum_{(l, m)} \sum_{n=0}^{\infty} \ln \left[\left(-p^{2}+\frac{(n+1 / 2+a / 2)^{2}}{R^{2}}\right)+\left(-p^{2}+\frac{(n+1 / 2-a / 2)^{2}}{R^{2}}\right)\right]+\sum_{(p, q)} \sum_{n=0}^{\infty} \ln \left(-p^{2}+\frac{(n+a / 2)^{2}}{R^{2}}\right) \\
& \left.+\sum_{p} \sum_{n=1}^{\infty} \ln \left(-p^{2}+\frac{(n-a / 2)^{2}}{R^{2}}\right)+3 \sum_{n=0}^{\infty} \ln \left(-p^{2}+\frac{n^{2}}{R^{2}}\right)+\sum_{n=0}^{\infty} \ln \left(-p^{2}+\frac{(n+a)^{2}}{R^{2}}\right)+\sum_{n=1}^{\infty} \ln \left(-p^{2}+\frac{(n-a)^{2}}{R^{2}}\right)\right],
\end{aligned}
$$

and for $A_{5}$ part is given by 


$$
\begin{aligned}
V_{\mathrm{eff}}^{A_{5}=} & -1 \frac{i}{2} \int \frac{d^{4} p}{(2 \pi)^{4}} \frac{1}{2 \pi R}\left[\sum_{i} \sum_{n=0}^{\infty} \ln \left(-p^{2}+\frac{n^{2}}{R^{2}}\right)+\sum_{j} \sum_{n=1}^{\infty} \ln \left(-p^{2}+\frac{n^{2}}{R^{2}}\right)+\sum_{k} \sum_{n=0}^{\infty} \ln \left(-p^{2}+\frac{(n+1 / 2)^{2}}{R^{2}}\right)\right. \\
& +\sum_{(l, m)} \sum_{n=0}^{\infty} \ln \left[\left(-p^{2}+\frac{(n+1 / 2+a / 2)^{2}}{R^{2}}\right)+\left(-p^{2}+\frac{(n+1 / 2-a / 2)^{2}}{R^{2}}\right)\right]+\sum_{(p, q)} \sum_{n=0}^{\infty} \ln \left(-p^{2}+\frac{(n+a / 2)^{2}}{R^{2}}\right) \\
& \left.+\sum_{p} \sum_{n=1}^{\infty} \ln \left(-p^{2}+\frac{(n-a / 2)^{2}}{R^{2}}\right)+3 \sum_{n=0}^{\infty} \ln \left(-p^{2}+\frac{n^{2}}{R^{2}}\right)+\sum_{n=0}^{\infty} \ln \left(-p^{2}+\frac{(n+a)^{2}}{R^{2}}\right)+\sum_{n=1}^{\infty} \ln \left(-p^{2}+\frac{(n-a)^{2}}{R^{2}}\right)\right],
\end{aligned}
$$

where $i=6,7,11,12,13,14,35, j=16, k=27,28,29,30,31,32,(l, m)=(1,19),(2,18),(4,21),(5,20),(9,23),(10,22)$, $(p, q)=(25,34),(26,33)$, and the last line terms come from $\left(B_{3}, B_{8}, B_{15}, B_{24}, B_{17}\right)$. We omit terms without $a$ dependences, since they have nothing to do with the dynamics of determining $a$. Then, we can obtain the VEV dependent effective potential as

$$
\begin{aligned}
V_{\mathrm{eff}}^{g+g h+A_{5}=} & -3 \frac{i}{2} \int \frac{d^{4} p}{(2 \pi)^{4}} \frac{1}{2 \pi R}\left[6 \sum_{n=-\infty}^{\infty} \ln \left(-p^{2}+\frac{(n+1 / 2-a / 2)^{2}}{R^{2}}\right)+2 \sum_{n=-\infty}^{\infty} \ln \left(-p^{2}+\frac{(n-a / 2)^{2}}{R^{2}}\right)\right. \\
& \left.+\sum_{n=-\infty}^{\infty} \ln \left(-p^{2}+\frac{(n-a)^{2}}{R^{2}}\right)\right] \\
= & -\frac{3}{2} C \sum_{n=1}^{\infty} \frac{1}{n^{5}}[6 \cos (\pi n(a-1))+2 \cos (\pi n a)+\cos (2 \pi n a)],
\end{aligned}
$$

where $C=3 / 64 \pi^{7} R^{5}$.

The SUSY version of the effective potential is easily obtained from this non-SUSY one. As in Ref. [4], it is obtained by replacing the coefficient $-2 / 3 C \rightarrow-2 C$ due to the change of degrees of freedom, and adding the factor (1 $-\cos (2 \pi n \beta))$ to $V_{\mathrm{eff}}^{\text {gauge }}$, where $\beta$ is the parameter of SS SUSY breaking.

\section{APPENDIX B: THE DERIVATION OF $V_{\text {eff }}^{m}$} by

The effective potential induced from bulk fields is given

$$
\begin{gathered}
V_{\text {eff }}\left[A^{0}\right]^{\text {fermion }}=f(D) \frac{i}{2} \operatorname{Tr} \ln D_{L}^{0} D^{0 L}, \\
V_{\text {eff }}\left[A^{0}\right]^{\text {scalar }}=-2 \frac{i}{2} \operatorname{Tr} \ln D_{L}^{0} D^{0 L},
\end{gathered}
$$

where $f(D)=2^{[D / 2]}$. The $V_{\text {eff }}^{m}$ can be calculated similar to the $V_{\mathrm{eff}}^{\text {gauge }}$. We introduce a fluctuation field $B$ according to the representation of bulk fields in Eqs. (B1) and (B2) as Eq. (A5), and calculating the eigenvalues. Here, we show only eigenvalues both in $S U(3)_{c} \times S U(3)_{W}$ and $S U(6)$ models.

In $S U(3)_{c} \times S U(3)_{W}$ model, adjoint representation fields with $\eta \eta^{\prime}=+$ have eigenvalues, $2 \times\left(n^{2} / R^{2}\right), \quad[(n$ $\left.\pm a)^{2} / R^{2}\right]$, and $2 \times\left[(n \pm a / 2)^{2} / R^{2}\right]$. Thus, $N_{a}^{(+)}$numbers of Dirac fermion induce $2 N_{a}^{(+)} C \sum\left(1 / n^{5}\right) \quad[\cos (2 \pi n a)$ $+2 \cos (\pi n a)]$. The adjoint representation fields with $\eta \eta^{\prime}$
$=-$ have eigenvalues, $2 \times\left[(n+1 / 2)^{2} / R^{2}\right], \quad[(n \pm a$ $\left.+1 / 2)^{2} / R^{2}\right]$, and $2 \times\left[(n \pm a / 2+1 / 2)^{2} / R^{2}\right]$. Thus, $N_{a}^{(-)}$numbers of Dirac fermion induce $2 N_{a}^{(-)} C \Sigma\left(1 / n^{5}\right)[\cos (2 \pi n(a$ $-1 / 2))+2 \cos (\pi n(a-1))]$. The fundamental representation fields with $\eta \eta^{\prime}=+$ have eigenvalues, $n^{2} / R^{2}$ and $(n$ $\pm a)^{2} / R^{2}$. Then $N_{f}^{(+)}$and $N_{s}^{(+)}$numbers of Dirac fermion and complex scalar induce $\left(2 N_{f}^{(+)}\right.$ $\left.-N_{s}^{(+)}\right) C \Sigma\left(1 / n^{5}\right) \cos (\pi n a)$. The fundamental representation fields with $\eta \eta^{\prime}=-$ have eigenvalues, $(n+1 / 2)^{2} / R^{2}$ and $(n \pm a+1 / 2)^{2} / R^{2}$. Then $N_{f}^{(-)}$and $N_{s}^{(-)}$numbers of Dirac fermion and complex scalar induce $\left(2 N_{f}^{(-)}\right.$ $\left.\left.-N_{s}^{(-)}\right) C \sum\left(1 / n^{5}\right) \cos (\pi n(a-1))\right]$.

In $S U(6)$ model, adjoint representation fields with $\eta \eta^{\prime}$ $=+$ have eigenvalues, $11 \times\left(n^{2} / R^{2}\right), 6 \times\left[(n+1 / 2)^{2} / R^{2}\right]$, $\left[(n \pm a)^{2} / R^{2}\right], \quad 2 \times\left[(n \pm a / 2)^{2} / R^{2}\right], \quad$ and $6 \times[(n \pm a / 2$ $\left.+1 / 2)^{2} / R^{2}\right]$. Thus, $N_{a}^{(+)}$numbers of Dirac fermion induce $2 N_{a}^{(+)} C \Sigma\left(1 / n^{5}\right)[6 \cos (\pi n(a-1))+2 \cos (\pi n a)+\cos (2 \pi n a)]$. The adjoint representation fields with $\eta \eta^{\prime}=-$ have eigenvalues, $\quad 11 \times\left[(n+1 / 2)^{2} / R^{2}\right], \quad 6 \times\left(n^{2} / R^{2}\right), \quad[(n \pm a$ $\left.+1 / 2)^{2} / R^{2}\right], \quad 2 \times\left[(n \pm a / 2+1 / 2)^{2} / R^{2}\right], \quad$ and $\quad 6 \times[(n$ $\left.\pm a / 2)^{2} / R^{2}\right]$. Thus, $N_{a}^{(-)}$numbers of Dirac fermion induce $2 N_{a}^{(-)} C \sum\left(1 / n^{5}\right)[6 \cos (\pi n a)+2 \cos (\pi n(a-1))+\cos (2 \pi n(a$ $-1 / 2))]$. The fundamental representation fields with $\eta \eta^{\prime}$ $=+$ have eigenvalues, $n^{2} / R^{2}, 3 \times\left[(n+1 / 2)^{2} / R^{2}\right], \quad[(n$ $\left.\pm a)^{2} / R^{2}\right]$. Then $N_{f}^{(+)}$and $N_{s}^{(+)}$numbers of Dirac fermion and complex scalar induce $\left(2 N_{f}^{(+)}\right.$ $\left.-N_{s}^{(+)}\right) C \sum\left(1 / n^{5}\right) \cos (\pi n a)$. The fundamental representation 
fields with $\eta \eta^{\prime}=-$ have eigenvalues, $(n+1 / 2)^{2} / R^{2}, 3$ $\times\left(n^{2} / R^{2}\right),\left[(n \pm(a-1))^{2} / R^{2}\right]$. Then $N_{f}^{(-)}$and $N_{s}^{(-)}$numbers of Dirac fermion and complex scalar induce $\left(2 N_{f}^{(-)}\right.$ $\left.\left.-N_{s}^{(-)}\right) C \sum\left(1 / n^{5}\right) \cos (\pi n(a-1))\right]$.
The effective potential in the SUSY case with SS breaking can also be obtained as in Ref. [4]. We add the factor $(1-\cos (2 \pi n \beta))$ in the effective potential induced from the fermion (ordinary particle) contributions.
[1] See, for examples, Y. Kawamura, Prog. Theor. Phys. 103, 613 (2000); 105, 691 (2001); 105, 999 (2001); G. Altarelli and F. Feruglio, Phys. Lett. B 511, 257 (2001); L. J. Hall and Y. Nomura, Phys. Rev. D 64, 055003 (2001); 65, 125012 (2002); 66, (2002); A. Hebecker and J. March-Russell, Nucl. Phys. B625, 128 (2002); B613, 3 (2001); A. B. Kobakhidze, Phys. Lett. B 514, 131 (2001); Y. Nomura, D. Smith, and N. Weiner, Nucl. Phys. B613, 147 (2001); R. Barbieri, L. J. Hall, and Y. Nomura, Phys. Rev. D 66, 045025 (2002); Nucl. Phys. B624, 63 (2002); N. Haba, T. Kondo, Y. Shimizu, T. Suzuki, and K. Ukai, Prog. Theor. Phys. 106, 1247 (2001); Y. Nomura, Phys. Rev. D 65, 085036 (2002); R. Dermisek and A. Mafi, ibid. 65, 055002 (2002); T. Li, Nucl. Phys. B619, 75 (2001); Phys. Lett. B 520, 377 (2001); L. J. Hall, Y. Nomura, and D. Smith, Nucl. Phys. B639, 307 (2002); T. Asaka, W. Buchmüller, and L. Covi, Phys. Lett. B 523, 199 (2001); L. J. Hall, Y. Nomura, T. Okui, and D. Smith, Phys. Rev. D 65, 035008 (2002); R. Barbieri, L. Hall, G. Marandella, Y. Nomura, T. Okui, S. Oliver, and M. Papucci, Nucl. Phys. B663, 141 (2003); L. J. Hall, J. March-Russell, T. Okui, and D. Smith, hep-ph/0108161; N. Haba, Y. Shimizu, T. Suzuki, and K. Ukai, Prog. Theor. Phys. 107, 151 (2002); N. Haba, T. Kondo, and Y. Shimizu, Phys. Lett. B 531, 245 (2002); 535, 271 (2002).

[2] D. B. Kaplan, H. Georgi, and S. Dimopoulos, Phys. Lett. B 136, 187 (1984); S. Dimopoulos and D. E. Kaplan, ibid. 531, 127 (2002).

[3] H. D. Kim, J. E. Kim, and H. M. Lee, J. High Energy Phys. 0206, 048 (2002); W. F. Chang and J. N. Ng, Phys. Rev. D 69, 056005 (2004).

[4] N. Haba, M. Harada, Y. Hosotani, and Y. Kawamura, Nucl. Phys. B657, 169 (2003).

[5] N. Haba, Y. Hosotani, and Y. Kawamura, Prog. Theor. Phys. 111, 265 (2004).

[6] N. S. Manton, Nucl. Phys. B158, 141 (1979); D. B. Fairlie, J. Phys. G 5, L55 (1979); Phys. Lett. B 82, 97 (1979).

[7] Y. Hosotani, Phys. Lett. B 126, 309 (1983); Ann. Phys. (N.Y.) 190, 233 (1989); Phys. Lett. B 129, 193 (1984); Phys. Rev. D 29, 731 (1984).

[8] N. V. Krasnikov, Phys. Lett. B 273, 246 (1991); H. Hatanaka, T. Inami, and C. S. Lim, Mod. Phys. Lett. A 13, 2601 (1998); G. R. Dvali, S. Randjbar-Daemi, and R. Tabbash, Phys. Rev. D 65, 064021 (2002); N. Arkani-Hamed, A. G. Cohen, and H.
Georgi, Phys. Lett. B 513, 232 (2001); I. Antoniadis, K. Benakli, and M. Quiros, New J. Phys. 3, 20 (2001).

[9] M. Kubo, C. S. Lim, and H. Yamashita, Mod. Phys. Lett. A 17, 2249 (2002).

[10] L. J. Hall, Y. Nomura, and D. R. Smith, Nucl. Phys. B639, 307 (2002).

[11] G. Burdman and Y. Nomura, Nucl. Phys. B656, 3 (2003).

[12] N. Haba and Y. Shimizu, Phys. Rev. D 67, 095001 (2003).

[13] I. Gogoladze, Y. Mimura, and S. Nandi, Phys. Lett. B 560, 204 (2003); 562, 307 (2003).

[14] C. Csaki, C. Grojean, H. Murayama, L. Pilo, and J. Terning, Phys. Rev. D 69, 055006 (2004).

[15] I. Gogoladze, Y. Mimura, S. Nandi, and K. Tobe, Phys. Lett. B 575, 66 (2003); K. Choi, N. Haba, K. S. Jeong, K. I. Okumura, Y. Shimizu, and M. Yamaguchi, J. High Energy Phys. 0402, 037 (2004).

[16] K. R. Dienes, E. Dudas, and T. Gherghetta, Phys. Lett. B 436, 55 (1998); Nucl. Phys. B537, 47 (1999).

[17] N. Arkani-Hamed, A. G. Cohen, and H. Georgi, hep-th/0108089.

[18] Th. Kaluza, Sitzungsber. K. Preuss. Akad. Wiss. 1921, 966 (1921); O. Klein, Z. Phys. 37, 895 (1926).

[19] J. Scherk and J. H. Schwarz, Phys. Lett. B 82, 60 (1979); Nucl. Phys. B153, 61 (1979).

[20] P. Fayet, Phys. Lett. B 159, 121 (1985); Nucl. Phys. B263, 649 (1986).

[21] I. Antoniadis, Phys. Lett. B 246, 377 (1990); I. Antoniadis, C. Munoz, and M. Quiros, Nucl. Phys. B B397, 515 (1993); A. Pomarol and M. Quiros, Phys. Lett. B 438, 255 (1998); I. Antoniadis, S. Dimopoulos, A. Pomarol, and M. Quiros, Nucl. Phys. B544, 503 (1999); A. Delgado, A. Pomarol, and M. Quiros, Phys. Rev. D 60, 095008 (1999).

[22] G. V. Gersdorff, M. Quiros, and A. Riotto, Nucl. Phys. B634, 90 (2002); G. V. Gersdorff and M. Quiros, Phys. Rev. D 65, 064016 (2002).

[23] K. Takenaga, Phys. Lett. B 570, 244 (2003).

[24] K. Takenaga, Phys. Rev. D 64, 066001 (2001).

[25] C. A. Scrucca, M. Serone, and L. Silvestrini, Nucl. Phys. B669, 128 (2003).

[26] C. Csaki, C. Grojean, and H. Murayama, Phys. Rev. D 67, 085012 (2003). 